\title{
Injection-seeded, gain-switched tunable Cr:ZnSe laser
}

\author{
Huseyin Cankaya, ${ }^{1}$ M. Natali Cizmeciyan, ${ }^{1}$ Ersen Beyatlı, ${ }^{1}$ A. Tolga Gorgulu, ${ }^{1}$ \\ Adnan Kurt, ${ }^{2}$ and Alphan Sennaroglu, ${ }^{1, *}$ \\ ${ }^{1}$ Laser Research Laboratory, Department of Physics, Department of Electrical-Electronics Engineering, Koç University, \\ Rumelifeneri, Sariyer, Istanbul 34450, Turkey \\ ${ }^{2}$ Teknofil, Inc., Zekeriyakoy, 34450, Istanbul, Turkey \\ *Corresponding author: asennar@ku.edu.tr
}

Received November 16, 2011; revised November 16, 2011; accepted November 17, 2011; posted November 18, 2011 (Doc. ID 151600); published January 9, 2012

\begin{abstract}
We report a narrow-linewidth, tunable, gain-switched Cr:ZnSe laser operating between 2255 and $2455 \mathrm{~nm}$. The spectral width of the laser was reduced from $125 \mathrm{~nm}$ to $0.3 \mathrm{~nm}$ by using injection seeding. Seeding was achieved with a second tunable CW Cr:ZnSe laser. The output wavelength was varied by tuning the wavelength of the seed laser. The seeded oscillator produced as high as $157 \mu \mathrm{J}$ pulses with $598 \mu \mathrm{J}$ incident pump pulse energy at a repetition rate of $1 \mathrm{kHz}$. The slope efficiency was determined to be $26 \%$. (c) 2012 Optical Society of America

OCIS codes: $140.3070,140.3538,140.3580,140.3600,140.3425$.
\end{abstract}

Cr:ZnSe lasers with broad tunability in the $2-3 \mu \mathrm{m}$ range find a multitude of applications in the pumping of optical parametric oscillators in the mid-IR [1] , in the generation of frequency combs for vibrational spectroscopy of molecules [2], and in remote sensing [3] . All of these applications require a coherent light source that has a well defined spectral output and that is tunable. Because of the high stimulated emission cross section, four-level energy structure, and absence of excited-state absorption in Cr:ZnSe, it is quite straightforward to obtain efficient lasing with a low threshold [ $\underline{4}-\underline{8}]$. However, especially during gain-switched operation where the gain far exceeds the losses, this also leads to simultaneous lasing over a very broad spectral bandwidth (of the order of tens of nanometers, often exceeding $100 \mathrm{~nm}$ ) and limits the effective use of the laser in the applications mentioned above. One approach to remedy this problem is via the use of intracavity wavelength-selective elements such as diffraction gratings [9-10]. However, the additional insertion loss introduced by the diffraction grating increases the lasing threshold and limits the available output power from the oscillator. An alternative approach employs injection seeding, whereby the spectral characteristics of the gain-switched laser are controlled by the seed laser having a narrower spectrum. Since the seed laser is coupled into the oscillator via an end mirror, no intracavity wavelength-selective elements are needed and no additional losses are introduced. Furthermore, if the seed source is tunable, it is possible to tune the output wavelength of the oscillator over the available bandwidth of the gain medium.

In this Letter, we describe an injection-seeded, gainswitched Cr:ZnSe laser with considerably improved spectral brightness. After employing injection seeding, the output linewidth (FWHM) of the oscillator was reduced from $125 \mathrm{~nm}$ to $0.3 \mathrm{~nm}$. As the seed light source, we used a second tunable continuous-wave (CW) Cr:ZnSe laser. By tuning the seed Cr:ZnSe laser with an intracavity prism, the output wavelength of the injection-seeded laser could be varied from 2255 to $2455 \mathrm{~nm}$. The gainswitched laser produced as high as $157 \mu \mathrm{J}$ of output energy at a wavelength of $2395 \mathrm{~nm}$ with $598 \mu \mathrm{J}$ of incident pump energy (pump wavelength of $1570 \mathrm{~nm}$ ) at a repetition rate of $1 \mathrm{kHz}$.
Figure 1 shows the experimental setup of the injectionseeded, gain-switched Cr:ZnSe laser. As the seed laser, we used a CW tunable Cr:ZnSe laser pumped by a Tm:fiber laser. The resonator of the CW seed laser consisted of two curved mirrors, each with a radius of curvature of $10 \mathrm{~cm}$ (M1 and M2), a flat end high reflector (M3), and a flat 3\% transmitting output coupler (OC1). A $2.5 \mathrm{~mm}$ long Cr:ZnSe crystal (C1) was positioned between M1 and M2 at Brewster incidence. The crystal had a small-signal absorption of $94 \%$ at a pump wavelength of $1800 \mathrm{~nm}$. The crystal was surrounded with indium foil and clamped between two copper holders whose temperature was kept at $21{ }^{\circ} \mathrm{C}$ by water cooling. The pump beam was focused to an estimated beam waist of $23 \mu \mathrm{m}$ inside the crystal with a convex lens (L1, focal length $=7.5 \mathrm{~cm}$ ). A Brewster-cut $\mathrm{CaF}_{2}$ prism $(\mathrm{P})$ was placed in the high reflector arm of the resonator to tune the output. An adjustable slit (S) was positioned between the prism and the end high reflector to reduce

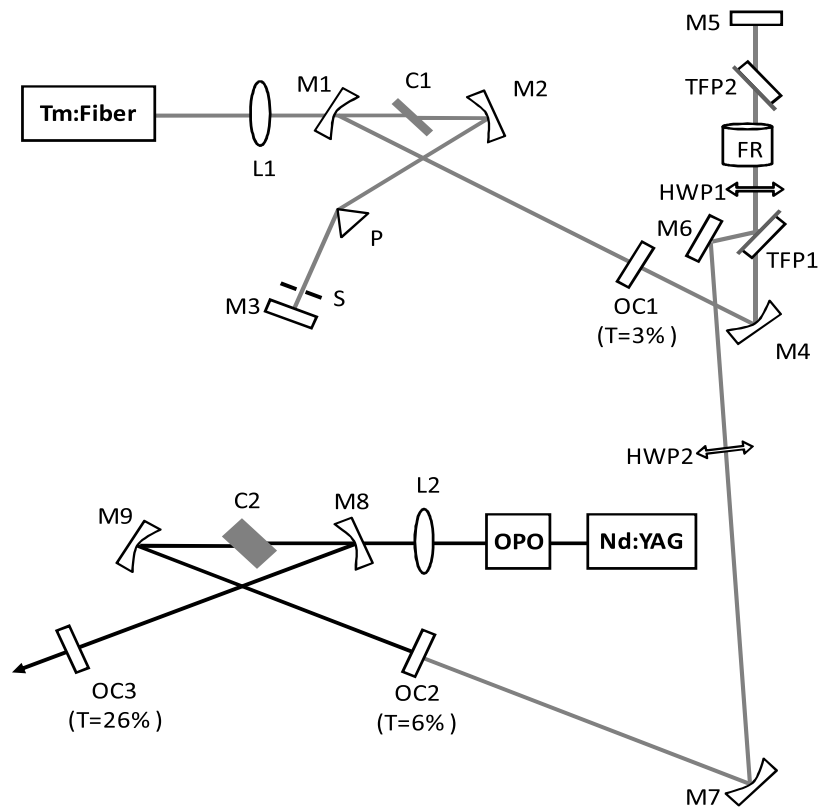

Fig. 1. Schematic of the injection-seeded, gain-switched tunable Cr:ZnSe laser. 
the spectral width of the seed laser. The high reflector and output coupler arm lengths were 65.5 and $83 \mathrm{~cm}$, giving an estimated laser waist of $35 \mu \mathrm{m}$ near the center of the stability region. The total cavity length of the seed laser was $160 \mathrm{~cm}$, corresponding to a free spectral range of $93.8 \mathrm{MHz}$. The output of the $\mathrm{CW}$ seed laser was collimated with a curved mirror (M4, radius of curvature $=2 \mathrm{~m}$ ) and passed through a Faraday isolator to protect the $\mathrm{CW}$ Cr:ZnSe laser from backreflection as well as from pulses originating from the gain-switched laser. The Faraday isolator consisted of two thin-film polarizers (TFP1 and TFP2), a half-wave plate (HWP1), a Faraday rotator (FR), and a flat aluminum retroreflecting mirror (M5).

The output of the isolator was then directed with a flat aluminum mirror (M6) to the gain-switched oscillator. A curved high reflector $(\mathrm{M} 7$, radius of curvature $=4 \mathrm{~m}$ ) was further used to keep the seed beam collimated. The estimated spot size of the seed laser inside the gain-switched Cr:ZnSe laser crystal was about $22 \mu \mathrm{m}$. With a second half-wave plate (HWP2), the polarization of the seed beam was adjusted to match the polarization of the beam emerging from the gain-switched oscillator. The $x$-type resonator of the gain-switched laser consisted of two curved mirrors (M8 and M9, radius of curvature $=20 \mathrm{~cm}$ ) and two flat output couplers having transmission values of $6 \%$ and $26 \%$ (OC2 and OC3 in Fig. 1 , respectively). The output coupler OC2 was used to couple the CW seed beam into the gain-switched resonator while the injection-seeded output was taken from OC3. The arm lengths of the resonator were 64 and $71 \mathrm{~cm}$, giving a beam waist of around $80 \mu \mathrm{m}$ in the gain medium. The total length of the cavity was $157 \mathrm{~cm}$, corresponding to a cavity mode spacing of $95.5 \mathrm{MHz}$. Inside the gainswitched resonator, a $6.6 \mathrm{~mm}$ long polycrystalline Cr:ZnSe (C2) sample was used at Brewster incidence. The crystal had a small-signal absorption of $92 \%$ at a pump wavelength of $1570 \mathrm{~nm}$. Because the absorption coefficient at $1570 \mathrm{~nm}$ is lower than that at $1800 \mathrm{~nm}$, a longer gain medium was used in the gain-switched oscillator to obtain a similar level of pump absorption. The gain-switched Cr:ZnSe oscillator was pumped by a $1570 \mathrm{~nm}$ optical parametric oscillator (OPO), which, in turn, was pumped by a $Q$-switched Nd:YAG laser at $1064 \mathrm{~nm}$. The pump pulsewidth (FWHM) and the pulse repetition rate were $50 \mathrm{~ns}$ and $1 \mathrm{kHz}$, respectively. The OPO pump beam was focused inside the crystal with a lens (L2) having a focal length of $20 \mathrm{~cm}$. By using the knife-edge technique, the pump beam waist was determined to be $90 \mu \mathrm{m}$. During the experiments, the spectrum of the gain-switched pulses was measured with a $0.5 \mathrm{~m}$ Czerny-Turner-type monochromator that had a resolution of $0.05 \mathrm{~nm}$.

Figure 2 shows the spectra of the unseeded and seeded gain-switched Cr:ZnSe laser for three different seed wavelengths on linear and logarithmic scales. In the absence of the CW seed laser, the gain-switched oscillator had an output spectral width (FWHM) of $125 \mathrm{~nm}$ centered around $2360 \mathrm{~nm}$. When the $\mathrm{CW}$ laser beam with a bandwith (FWHM) of $0.3 \mathrm{~nm}$ was injected into the gainswitched laser cavity, the spectral width of the gainswitched laser was narrowed down to about $0.3 \mathrm{~nm}$, corresponding to a bandwidth of $16 \mathrm{GHz}$. In other words,

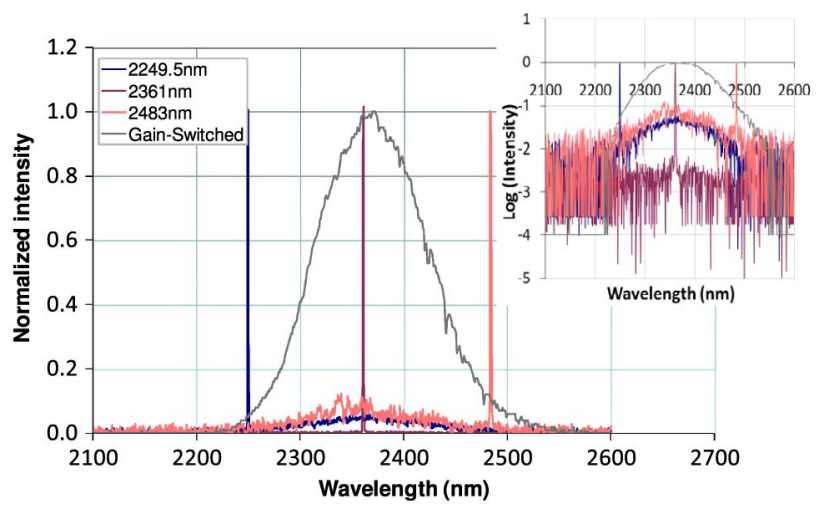

Fig. 2. (Color online) Spectra of the unseeded and injectionseeded gain-switched Cr:ZnSe laser on linear and logarithmic (inset) scales.

the spectral brightness of the laser was improved by a factor of 417 . For both resonators, there were approximately 350 simultaneously oscillating cavity modes during injection seeding. In the experiments, we observed a strong dependence of the linewidth of the injectionseeded laser on the CW laser spectrum. In the absence of an adjustable slit inside the CW laser cavity, the linewidth of the CW laser was broader, and spectral hopping became very significant. In this case, the spectral linewidth of the injection-seeded laser increased to around $2 \mathrm{~nm}$, covering approximately 2300 longitidunal modes, and additional parasitic spikes on the spectrum could be observed. By adjusting the slit width to reduce the linewidth of the CW laser to about $0.3 \mathrm{~nm}$, we could eliminate the parasitic spikes and spectral jitter in the output of the gain-switched laser. During the spectrum measurements, with $600 \mu \mathrm{J}$ of input pulse energy, the laser output energy varied between 100 and $130 \mu \mathrm{J}$, depending on the wavelength of the seed laser. For example, with incident CW seed power of $0.8,5$, and $1 \mathrm{~mW}$ at wavelengths of 2249.5, 2361, and $2483 \mathrm{~nm}$, the gain-switched laser output pulse energy was 108,130 , and $120 \mu \mathrm{J}$, respectively. Below $1 \mathrm{~mW}$ of seed power, the residual unseeded spectrum reapperead. The $M^{2}$ parameter of the seeded laser was further determined with the knife-edge method to be 2.03 .

The wavelength of the injection-seeded laser could be varied in the range of $2250-2483 \mathrm{~nm}$ by tuning the CW seed laser. When the CW laser was tuned to the two edges of the unseeded gain-switched laser spectrum, the residual unseeded laser spectrum also reappeared (see Fig. 2). The tuning range was limited by the reflectivity bandwidth of the cavity optics. Figure 3 further shows the tuning curve of the injection-seeded $\mathrm{Cr}: \mathrm{ZnSe}$ laser between 2255 and $2455 \mathrm{~nm}$. In this spectral range, the residual unseeded gain-switched laser spectrum was not present. The measurements were made at an incident pump energy of $600 \mu \mathrm{J}$. The highest pulse energy was obtained at $2360 \mathrm{~nm}$, which was also the wavelength of peak emission from the unseeded gain-switched Cr:ZnSe laser. For both seeded and unseeded cases, the temporal pulse duration of the laser was measured to be around $30 \mathrm{~ns}$.

Figure 4 shows the efficiency curves of the unseeded and injection-seeded Cr:ZnSe laser. The output pulse 


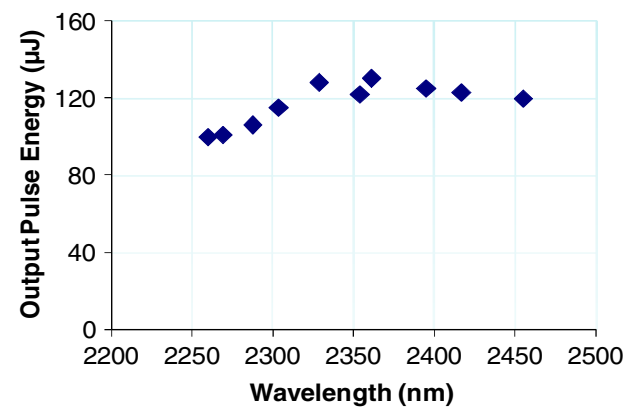

Fig. 3. (Color online) Tuning curve of the injection-seeded, gain-switched Cr:ZnSe laser. By tuning the CW seed laser, a narrow-line output could be obtained from the seeded Cr:ZnSe laser between 2255 and $2455 \mathrm{~nm}$.

energy was measured after the $26 \%$ output coupler (OC3). The slope efficiency of the unseeded GS laser was determined to be $22.5 \%$ with respect to the incident pump energy. By comparing the slope efficiencies at different output coupling levels, the passive loss of the cavity was further found to be $5.6 \%$, primarily due to losses in the gain medium. Above $480 \mu \mathrm{J}$ of incident pump energy, saturation was observed in the output energy of the unseeded oscillator. This is due to the depletion of the ground-state population, resulting in a reduction of the pump absorption. However, in the case of the injectionseeded laser, this saturation effect was observed at a higher incident pump energy ( $598 \mu \mathrm{J})$. In the case of injection seeding, gain-switched laser pulses evolve from the seed laser light rather than noise. Hence, this reduces the time required for pulse formation. As a result, a higher rate of stimulated emission, initiated by the evolving laser pulse, replenishes the ground-state population more effectively and reduces the saturation of pump absorption.

In summary, we described a proof-of-principle experiment to reduce the spectral linewidth of a gain-switched Cr:ZnSe laser by CW injection seeding. An important advantage of the proposed scheme is that intracavity wavelength-selective elements are not introduced into the gain-switched oscillator, and hence no additional losses are introduced. Furthermore, because the saturation of the pump absorption is reduced, higher output energy can be obtained from the oscillator in comparison with the unseeded case. In the experiments, the seeded Cr:ZnSe oscillator could be tuned between 2255 and $2455 \mathrm{~nm}$ by tuning the $\mathrm{CW}$ seed laser with an intracavity prism. The linewidth of the laser was further reduced from 125 to $0.3 \mathrm{~nm}$ during injection seeding. The laser could produce as high as $157 \mu \mathrm{J}$ pulses with $598 \mu \mathrm{J}$ of incident pump energy at a $1 \mathrm{kHz}$ repetition rate. In

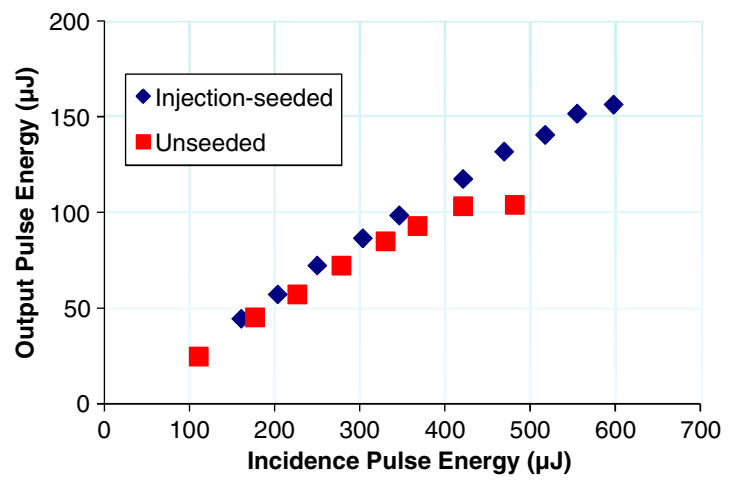

Fig. 4. (Color online) Efficiency curve of the injection-seeded and unseeded Cr:ZnSe laser as a function of the incident pump pulse energy.

principle, the spectral brightness of the seeded laser can be further improved by introducing more wavelength-selective elements into the seed laser, such as etalons.

We thank R. Kortan for donating some of the equipment and U. Demirbas for useful discussion. This project was supported by TUBITAK, the Scientific and Technological Council of Turkey, under grant 108T028.

\section{References}

1. W. S. Pelouch, G. J. Wagner, T. J. Carrig, and W. J. Scharpf, in Advanced Solid-State Lasers, S. Payne and C. Marshall, eds., Vol. 46 of OSA Trends in Optics and Photonics (Optical Society of America, 2001), pp. 670-674.

2. B. Bernhardt, E. Sorokin, P. Jacquet, R. Thon, T. Becker, I. T. Sorokina, N. Picque, and T. W. Hansch, Appl. Phys. B 100, 3 (2010).

3. C. Fischer, E. Sorokin, I. T. Sorokina, and M. W. Sigrist, Opt. Lasers Eng. 43, 573 (2005).

4. H. Cankaya, U. Demirbas, A. K. Erdamar, and A. Sennaroglu, J. Opt. Soc. Am. B 25, 794 (2008).

5. L. D. DeLoach, R. H. Page, G. D. Wilke, S. A. Payne, and W. F. Krupke, IEEE J. Quantum Electron. 32, 885 (1996).

6. U. Demirbas and A. Sennaroglu, Opt. Lett. 31, 2293 (2006).

7. R. H. Page, K. I. Schaffers, L. D. DeLoach, G. D. Wilke, F. D. Patel, J. B. Tassano Jr., S. A. Payne, W. F. Krupke, K.-T. Chen, and A. Burger, IEEE J. Quantum Electron. 33, 609 (1997).

8. I. S. Moskalev, V. V. Fedorov, and S. B. Mirov, Opt. Express 16, 4145 (2008).

9. G. J. Wagner, B. G. Tiemann, W. J. Alford, and T. J. Carring, in Advanced Solid-State Photonics (TOPS), G. Quarles, ed., Vol. 94 of OSA Trends in Optics and Photonics (Optical Society of America, 2004), paper 371.

10. C. H. Zhang, P. B. Meng, B. Q. Yao, G. Li, Y. L. Ju, and Y. Z. Wang, Laser Phys. 21, 44 (2011). 